\title{
DIEZ AÑOS DE MEDICINA BASADA EN PRUEBAS. INTRODUCCIÓN A LA UROLOGÍA BASADA EN PRUEBAS
}

\author{
C. TELLO ROYLOA
}

Grupo de Trabajo en Urología Basada en Pruebas de la Asociación Murciana de Urología*.

Actas Urol Esp. 27 (4): 251-259, 2003

\section{RESUMEN}

\section{"DIEZ AÑOS DE MEDICINA BASADA EN PRUEBAS. INTRODUCCIÓN A LA UROLOGÍA} BASADA EN PRUEBAS"

La Medicina Basada en la Evidencia o Medicina Basada en Pruebas (MBP) se ha convertido, en sus diez años de existencia, en un fenómeno social. La MBP ha sido definida como: el uso consciente explícito y juicioso de las mejores pruebas disponibles en la toma de decisiones sobre el cuidado del paciente individual. El análisis crítico de la atención sanitaria, la incorporación de nuevos métodos de investigación clínica y el desarrollo de la informática y la comunicación pueden considerarse las bases para la aparición de la MBP y otros movimientos relacionados, tales como la Evaluación de Tecnologías Sanitarias, el programa de habilidades en lectura crítica o la Colaboración Cochrane. La MBP es un sistema de aprendizaje autodidacta y permanente, que consiste en la ejecución sucesiva de los siguientes puntos: 1) la conversión de las necesidades de información en preguntas contestables, 2) la obtención de las mejores pruebas, 3) la revisión crítica de esas pruebas en cuanto a su validez, importancia y aplicabilidad y 4) la aplicación de los resultados. El modelo de MBP ha recibido también críticas y objeciones, que se analizan. La Asociación Murciana de Urología ha creado un Grupo de Trabajo para el estudio, la práctica y la aplicación de esta metodología en la especialidad de urología.

PALABRAS CLAVE: Medicina Basada en la Evidencia. Urología.

\begin{abstract}
"TEEN YEARS OF EVIDENCE-BASED MEDICINE. INTRODUCTION AT EVIDENCE-BASED UROLOGY"

The Evidence-based Medicine has become, in ten years of existence, a social phenomenon, that has spread to all the sectors of the health. The MBP has been defined as: the conscientious, explicit and judicious use of current best evidence in making decisions about the care of individual patients. The critical analysis of the health care, the new methods of clinical investigation and the development of computer science and the communication can be considerate the bases for the appearance of the MBP and other movements or related currents of thought, such as the Health Technology Assessment, the "Critical Appraisal Skils Programme" or the "Cochrane Collaboration". The MBP is a system of self-taught and permanent learning, that consists of the successive execution of the following points: 1) converting the need for information into an answerable question, 2) tracking down the best evidence with which to answer that question, 3) critically appraising that evidence for its validity, impact, and applicability, and 4) the application of the results actually clinical. The MBP model has also received critics and objections, that are analyzed. The "Asociación Murciana de Urología" has created a Working Group for the study, the practice and the application of this methodology in urology.
\end{abstract}

KEY WORDS. MESH TERMS: Evidence-based Medicine. Urology.

*Miembros del "Grupo de Trabajo en Urología Basada en Prueba de la Asociación Murciana de Urología”: C. Tello Royloa (Coordinador), L. Asensio Egea, E. Cao Avellaneda, T. Fernández Aparicio, L.O. Fontana Compiano, F. García García, P. Guzmán Martínez-Valls, G. Hita Villaplana, P. López Cubillana, B. Miñana López, M. Pérez Albacete, A. Prieto González, M. Rigabert Montiel, A. Romero Hoyuela, A. Sempere Gutiérrez, M. Tomás Ros, P. Valdelvira Nadal. 
$\mathrm{H}$ ace diez años, en noviembre de 1992, el "Evidence-Based Medicine Working Group" (EBMWG), Grupo de Trabajo en Medicina Basada en Pruebas, publicaba en la revista JAMA, el artículo titulado: "Evidence-based medicine. A new approach to teaching the practice of medicine ${ }^{\prime 1}$ (Medicina Basada en Pruebas. Un nuevo enfoque para la docencia de la práctica de la medicina ${ }^{2}$. Este trabajo, atrevido y provocador, proponía un cambio en el modelo o paradigma del aprendizaje y la práctica de la medicina y formulaba el ideario de la llamada Medicina Basada en Pruebas (MBP). Aunque un año antes Gordon Guyatt, presidente del Grupo, había publicado ya un editorial en la revista ACP Journal Club ${ }^{3}$ en el que se introducía el concepto de "Evidence-based medicine", el artículo de JAMA, fue en realidad la presentación en sociedad de este movimiento, iniciado en la década de los ochenta en la Universidad canadiense de Mc Master en Ontario.

La expresión "Evidence-based medicine" se ha traducido al español como Medicina Basada en la Evidencia. Esta traducción, ya casi consagrada, es sin embargo incorrecta y poco esclarecedora. En nuestro idioma la palabra evidencia (del latín evidentia, e-videre) tiene el significado de: "certeza clara, de la que no se puede dudar"4. El término inglés "evidence" tiene un sentido más amplio englobando conceptos como: prueba, hecho o indicio. Atendiendo a la idea, que sus promotores bautizaron con "Evidence-based medine", se han propuesto distintas traducciones, más explicativas, como medicina basada en la metodología científica racional correcta ${ }^{5}$, medicina basada en la revisión sistemática de la bibliografía o en la revisión crítica de la literatu$\mathrm{ra}^{6}$ o medicina basada en pruebas ${ }^{7}$ que es la aquí se ha adoptado.

La MBP se ha revelado como un auténtico fenómeno social, que se ha difundido con rapidez por todo el mundo y a todos los sectores de la sanidad, transcendiendo incluso el ámbito profesional $^{8,9}$. Un ejemplo de la importancia de este modelo es el hecho de que la National Library of Medicine (NLM) incluyera en 1997 el término "evidence-based medicine" en su vocabulario MeSH (Medical Subject Headings) y que desde entonces haya en Medline más de 400 citas indexadas bajo ese descriptor.

\section{DEFINICIÓN Y CONCEPTO DE LA MEDICINA BASADA EN PRUEBAS}

Durante sus primeros años de difusión, la expresión "Evidence-based medicine", se empleó sin que existiese ninguna definición explícita, lo que probablemente contribuyó al desconocimiento real de la propuesta y a variadas interpretaciones sobre su significado. De hecho, ni en el artículo inicial, ni en las posteriores "guías para usuarios de la literatura médica", publicadas en JAMA por el EBMWG, se definió explícitamente el concepto.

Años más tarde, en 1996, el Prof. David Sackett, su más conocido promotor, definió la Medicina Basada en Pruebas como el uso consciente, explícito y juicioso de las mejores pruebas disponibles en la toma de decisiones sobre el cuidado del paciente individual. Precisando, que practicar la MBP es integrar: la competencia o maestría individual, es decir la experiencia clínica, con las mejores pruebas externas disponibles, o lo que es lo mismo con los últimos hechos científicamente probados. Ambos aspectos: experiencia personal y aplicación de las "evidencias", deben ir unidos. Sin el conocimiento y la aplicación de las últimas pruebas disponibles el ejercicio de la medicina queda rápidamente desfasado con el consiguiente perjuicio para el paciente. Pero sin la experiencia clínica la medicina puede convertirse en un ejercicio automático y no menos perjudicial. Una maniobra diagnóstica o terapéutica de utilidad científicamente comprobada puede ser inapropiada para un paciente concreto ${ }^{10}$.

En esta definición, se resumen, sin duda, las dos grandes directrices de cualquier clínico actual: el valor de la experiencia clínica y la necesidad de "estar al día". Cabe entonces preguntarse, qué novedades aporta la MBP y por qué se propone como un concepto distinto, incluso revolucionario frente a la medicina "tradicional".

El EBMWG, estableció en su artículo inicial una comparación entre lo que denominaron el "antiguo paradigma" de la medicina y su "nuevo paradigma", el modelo de la MBP.

El esquema tradicional de la enseñanza y el ejercicio de la medicina están basados en el principio de autoridad, en la primacía de la experiencia clínica, en el conocimiento de las bases fisiopatológicas de la enfermedad y en un modelo paternalista de la relación médico-enfermo. 
La MBP disminuye la importancia de la intuición, de la experiencia clínica y de los principios fisiopatológicos como base para tomar las decisiones asistenciales, mientras que resalta la importancia de examinar los resultados que surgen de la investigación clínica. Reduce el valor de la autoridad y defiende la implicación activa del paciente en la toma de decisiones clínicas.

La pretensión del EBMWG de que la MBP sea considerada como un cambio de paradigma de la medicina, resulta excesivamente presuntuosa y ha sido ampliamente debatida ${ }^{11}$.

El verdadero punto de inflexión histórico en el modo de entender y practicar la medicina, se produjo en el siglo XIX, como consecuencia de la fundamentación de la medicina en las ciencias naturales y la explicación de las enfermedades como trastornos corporales estructurales y dinámicos que corresponden a causas naturales, específicas, objetivables y, consecuentemente, cuantificables. El nacimiento de esta nueva medicina se desarrolló en dos etapas sucesivas: la llamada "medicina hospitalaria" basada en el método anatomoclínico y la "medicina de laboratorio" fundamentada en las mentalidades fisiopatológica y etiopatogénica ${ }^{12}$.

El propio David Sackett, sitúa los orígenes filosóficos de la Medicina Basada en la Evidencia, en la Escuela de París, a mediados del siglo XIX ${ }^{10}$. Así pues, "Más que constituir una revolución, parece que la medicina basada en la evidencia no es sino un ejemplo de un nuevo avance y profesionalización del paradigma actual..."11.

Sin embargo, rechazar que la medicina basada en pruebas sea un nuevo paradigma, no disminuye en absoluto la importancia y el impacto que este fenómeno está teniendo en la enseñanza y en la práctica de la medicina occidental ${ }^{12}$.

La MBP es, en realidad, una metodología, un sistema de aprendizaje autodidacta y permanente que tiene su punto de partida y su destino en la atención diaria al paciente. La práctica de este nuevo modo de hacer medicina consiste en la ejecución sucesiva de los siguientes puntos:

1. La conversión de las necesidades de información, que surgen en la práctica diaria, en preguntas contestables.

2. La obtención de las mejores pruebas que respondan a las preguntas.
3. La revisión crítica de esas pruebas en cuanto a su validez y utilidad clínica.

4. La aplicación de los resultados en la práctica clínica.

\section{FUNDAMENTOS PARA UNA MEDICINA BASADA EN PRUE BAS}

El motivo de la aparición de la MBP, y de otros movimientos, corrientes de pensamiento o instituciones, precedentes o concurrentes, que más adelante se detallarán, puede tener su origen en una serie de hechos que se fueron produciendo durante las últimas décadas del pasado siglo XX. Entre ellos podríamos destacar:

1. El análisis crítico de la atención sanitaria, con la identificación de algunas contradicciones y problemas en la medicina y la sanidad.

2. El desarrollo de nuevos métodos de investigación clínica.

3. El acceso a la información favorecido por el desarrollo de la informática y las tecnologías de la comunicación.

\section{Análisis crítico de la atención sanitaria}

En las últimas décadas del siglo XX, desde distintos sectores de la sanidad: clínicos, investigadores, profesores o gestores, alertaron sobre la existencia de algunas contradicciones y problemas en el seno de la medicina: la constatación de una variabilidad inexplicable en la práctica clínica, el reconocimiento de que muchas actuaciones médicas no estaban basadas en hechos científicamente probados, la dificultad de los clínicos para acceder a la información científica relevante, la inexistencia de revisiones sistemáticas, la preocupación por el continuo crecimiento del gasto sanitario o la crisis del modelo paternalista en la relación médico-paciente.

Desde finales de los años sesenta y principios de los setenta un gran número de estudios han puesto de manifiesto la existencia de importantes variaciones en la práctica médica, que pueden explicarse por factores poblacionales o derivados de los sistemas sanitarios, pero también por la incertidumbre de los médicos ante la falta de "evidencias" científicas o su incapacidad para conocerlas $^{13}$.

Durante algunos años se manejó el dato, publicado por la desaparecida Office of Technology 
Assessment del Congreso de Estados Unidos en 1978 y 1983, de que sólo el 10-20\% de los procedimientos médicos habían demostrado científicamente su eficacia. Aunque posteriores estudios han elevado la cifra hasta el 69\%, sigue siendo un motivo de preocupación, el hecho de que algunas o muchas de las intervenciones médicas carezcan de una base científica constatada ${ }^{14,15}$.

La falta de conocimiento por parte de los médicos de pruebas aportadas por la investigación clínica, en las que sustentar sus decisiones, no implica siempre su inexistencia. La incapacidad de los clínicos para acceder a la información es un hecho reconocido. El enorme volumen de la literatura científica, su variable calidad y su mala organización son algunas de las dificultades que limitan el conocimiento de la información relevante $^{10}$.

En 1979 el epidemiólogo británico Archie Cochrane, expresó la necesidad de promover una iniciativa mundial, similar a la Colaboración que hoy lleva su nombre:".. ciertamente, se puede hacer una gran crítica a la profesión médica por no haber organizado un resumen crítico, por especialidad o subespecialidad, actualizado periódicamente, de todos los ensayos clínicos controlados que sean relevantes" 16 .

Aunque los promotores de la MBP, se desligan de posibles objetivos económicos ${ }^{17}$, la preocupación por el crecimiento del gasto sanitario y el interés por un uso adecuado de los recursos está presente en la medicina actual.

Finalmente, durante las últimas décadas, se han producido también notables cambios con respecto al papel del paciente en la toma de decisiones médicas. Este debate llevó a Ezekiel y Linda Emanuel a definir en 1992 sus ya clásicos modelos de la relación médico-paciente: paternalista, informativo, interpretativo y deliberativo ${ }^{18}$.

\section{Metodología de investigación clínica}

Paralelamente a esta identificación de problemas y conflictos en la medicina, durante la segunda mitad del siglo XX se produjo también el nacimiento y desarrollo de nuevos métodos de investigación clínica. El ensayo clínico introducido a principios de los años cincuenta por Sir Austin Bradford Hill y cuya metodología se ha desarrollado progresivamente durante más de 25 años, se ha convertido en herramienta imprescindible para determinar la eficacia y seguridad de los tratamientos. Han aparecido técnicas cualitativas y cuantitativas de síntesis de la información, como las revisiones sistemáticas y el meta-análisis. Se ha definido un nuevo modelo de documento que integra la información de los ensayos clínicos y de otro tipo de estudios empíricos: las guías de práctica clínica entendidas como: "recomendaciones desarrolladas sistemáticamente para ayudar a los médicos y a los pacientes a decidir sobre la atención sanitaria más apropiada en circunstancias clínicas concretas"19. La epidemiología poblacional ha aportado al estudio de los pacientes individuales algunos de sus métodos de investigación, creándose así una nueva especialidad: la epidemiología clínica ${ }^{9}$.

\section{Informática y comunicación}

Por último la introducción de la informática a la documentación científica, con la creación de bases de datos bibliográficas y textuales, y el desarrollo de las tecnologías de la comunicación, especialmente la popularización de Internet, está facilitado el acceso a la información científica. Como consecuencia de ello, se ha despertado entre clínicos e investigadores el deseo de disponer libremente de lo que consideramos nuestro patrimonio: la producción científica internacional. Así lo demuestran iniciativas como la Public Library of Science $^{20}$ (Biblioteca Pública de la Ciencia), que finalmente parece haber cristalizado en la creación por parte de la NLM de la base de datos PubMed Central $^{21}$.

\section{MEDICINA BASADA EN PRUEBAS Y MOVIMIENTOS LIMÍTROFES}

El resultado del análisis crítico de la atención sanitaria, de la incorporación de los nuevos métodos de investigación clínica, y de las tecnologías de la información y de la comunicación, ha sido la aparición de distintas corrientes de pensamiento, movimientos, organizaciones o grupos de trabajo, tales como: $\mathrm{El}$ análisis de resultados ("Outcomes research"), la Evaluación de Tecnologías Sanitarias, el programa de habilidades en lectura crítica ("Critical Appraisal Skills Programme"), la Colaboración Cochrane, o la propia Medicina Basada en Pruebas, ("Evidence based-medicine"), 
que intentan mejorar distintos aspectos de la práctica médica, y que están aportando un nuevo impulso a la medicina.

\section{Análisis de Resultados y Evaluación de Tecnologías Sanitarias}

Antes de la aparición de la MBP, habían surgido ya los conceptos del análisis de resultados ("Outcomes research") y de Evaluación de Tecnologias Sanitarias.

En 1989, se creó en Estados Unidos la Agency for Health Care Policy and Research, con la finalidad de financiar y apoyar la realización de estudios sobre los resultados (outcomes) de las intervenciones clínicas. Una de las iniciativas más importantes de esta agencia fue el desarrollo de los denominados Patient Outcomes Research Teams, equipos multidisciplinarios dedicados a analizar los resultados y costes de las distintas alternativas de tratamiento de enfermedades concretas, entre ellas la hiperplasia prostática benigna y el cáncer de próstata localizado.

En años sucesivos, con el impulso de las administraciones públicas de distintos países, han ido apareciendo distintas agencias dedicadas a la Evaluación de Tecnologías Sanitarias (ETS).

La Evaluación de Tecnologías Sanitarias, ha sido definida como: "aquel proceso de análisis e investigación, dirigido a estimar el valor y contribución relativos de cada tecnología sanitaria a la mejora de la salud individual y colectiva, teniendo además en cuenta su impacto económico y social" 22 .

En nuestro país se han ido creando en los últimos años: La Agencia de Evaluación de Tecnologías Sanitarias del Instituto Carlos III, la Agència d’Avaluació de Tecnología Mèdica de Cataluña, la Agencia de Evaluación de Tecnologías Sanitarias del País Vasco/Osasunerako Teknologien Ebaluaketa (OSTEBA), la Agencia de Evaluación de Tecnologías Sanitarias de Andalucía (AETSA), la Fundación Canaria de Investigación de la Salud (FUNDIS), y una Asociación Española de Evaluación de Tecnologías Sanitarias.

Todas estas Agencias están coordinadas en una red internacional: La "International Network of Agencies for Health Technology Assessment" (INAHTA) y una sociedad internacional: la
"International Society for Health Technology Assessment in Health Care" (ISHTAC), que mantienen bases de datos que reúnen los trabajos de las distintas agencias.

\section{La Colaboración Cochrane}

La Colaboración Cochrane, fundada en 1993, es una organización internacional, independiente $\mathrm{y} \sin$ animo de lucro, que busca ayudar a quienes necesitan tomar decisiones bien informadas mediante la preparación, actualización y divulgación de revisiones sistemáticas sobre los efectos de la atención sanitaria. El trabajo de la Colaboración Cochrane se divulga fundamentalmente a través de la Cochrane Library (Biblioteca Cochrane), una publicación periódica trimestral, en formato electrónico (CD-ROM o Internet), que contiene diversas bases de datos. La principal es la "Cochrane Database of Systematic Reviews CDSR", que contiene las revisiones sistemáticas elaboradas por los distintos Grupos de Revisores que integran la Colaboración Cochrane. La "Cochrane Controlled Trials Regiter - CCTR", es un registro de ensayos clínicos controlados recopilado por la Colaboración, en un importante esfuerzo de búsqueda electrónica y manual. Otras bases de datos de la Biblioteca Cochrane son: la "Cochrane Database of Abstracts of Reviews of Effectiveness - DARE" (Base de datos de resúmenes de revisiones de eficacia), producida por el Centro de Revisores de la Universidad de York o la "Cochrane Reviews Metodology Database", que contiene información sobre aspectos relacionados con la metodología de las revisiones ${ }^{23}$. Recientemente se ha editado la versión en español bajo el título: "Cochrane Library plus", que sólo está disponible a través de la red.

Programa de habilidades en lectura critica

(Clinical Appraisal Skills Programe. CASP)

El Programa de habilidades en lectura crítica (Clinical Appraisal Skills Programe. CASP), creado en 1993, es una iniciativa para ayudar a quienes tienen que tomar decisiones en el Servicio de Salud Inglés, a desarrollar la capacidad para leer críticamente los trabajos científicos ${ }^{24}$.

Derivado de CASP, el "Programa de habilidades en lectura críticas - España" (CASPe ${ }^{25}$ promueve la celebración de talleres sobre lectura crítica en 
distintos hospitales de nuestro país y forma parte de CASP-Internacional Network (CASPi) ${ }^{26}$, una red de colaboración internacional en la que participan distintos países europeos y americanos.

Todos estos movimientos representan en realidad soluciones y herramientas complementarias orientadas a un mismo fin. El funcionamiento de la actividad clínica diaria y de la gestión sanitaria, en sus distintos niveles, está determinada por una continua toma de decisiones. Los gestores, los clínicos o los propios pacientes tienen que tomar diariamente decisiones, según sus responsabilidades, acerca de la planificación de la política sanitaria, de la necesidad de implantar una nueva técnica diagnóstica o terapéutica en un hospital o en un servicio, sobre la conveniencia de utilizar determinado tratamiento en un paciente concreto o sobre la necesidad de someterse a dicho tratamiento. En cualquier caso, la toma de decisiones se debe basar siempre en la mejor información disponible. La fuente de documentación para obtener esta información es la bibliografía científica, que sin embargo, resulta a menudo inaccesible por su volumen. Los gestores, en sus distintos niveles, utilizan los informes proporcionados por las Agencias de Evaluación de Tecnologías Sanitarias para orientar sus "macro" y "meso-decisiones". Los clínicos, y los pacientes a través de la información que los médicos les proporcionen, deberán tomar sus "micro-decisiones" a partir de los datos obtenidos en la búsqueda y la valoración crítica de la bibliografía bio-médica, que es en esencia la metodología de la MBP. En este trabajo de búsqueda, evaluación y síntesis de las mejores "evidencias", que comparten la Evaluación de Tecnologías Sanitarias y la práctica de una MBP, son excelentes herramientas los trabajos de la Colaboración Cochrane y otras publicaciones secundarias.

\section{PRÁCTICA DE LA MEDICINA BASADA EN PRUEBAS}

La práctica de la MBP es la aplicación de una metodología de trabajo que ayuda al clínico a resolver las dudas con las que se encuentra frente al paciente concreto y a mantenerse al día.

La metodología de la MBP propone un esquema de trabajo en varios pasos sucesivos:

1. Formulación de preguntas contestables.

2. Obtención de las mejores pruebas.
3. Revisión crítica de las pruebas.

4. Aplicación de los resultados en la práctica clínica.

\section{Formulación de preguntas contestables}

El primer paso en la práctica de la MBP es la conversión de las necesidades de información que surgen en la práctica clínica en preguntas simples y claramente definidas, que reflejen el objetivo concreto de la investigación. La pregunta clínica, o si se quiere, el objetivo de la investigación, debe incluir y definir de forma explícita cuatro elementos: el paciente o problema, la intervención que se quiere considerar, ya sea una prueba diagnóstica, un tratamiento, un pronóstico..., la intervención con la se quiere comparar (si procede) y la variable o variables que miden el resultado ${ }^{10}$.

\section{Obtención de las mejores pruebas}

La obtención de las mejores pruebas externas disponibles supone la búsqueda y recuperación de los resultados de la investigación clínica. Estas pruebas, pueden encontrarse en dos tipos de documentos: la literatura primaria, aquella que expone por primera vez descubrimientos científicos, observaciones originales o resultados de la investigación experimental y clínica y la literatura secundaria, aquella que reseña la información publicada por las fuentes primarias.

Los problemas a los que se enfrenta el clínico en la búsqueda de información son: el enorme volumen que ha alcanzado la literatura científica, su mala organización y su variable calidad. La práctica de la MBP requiere el conocimiento de aquellos recursos que permiten acceder de forma rápida a una información actual, concreta y de calidad. Es necesario saber: dónde y cómo buscar. La informática aplicada a la documentación científica ha hecho posible la creación de grandes bases de datos bibliográficos y textuales, que gracias al desarrollo de las tecnologías de la comunicación, resultan accesibles a través de Internet. Pero la realización de búsquedas bibliográficas, para ser eficaz, requiere de algunos conocimientos básicos sobre documentación científica, como el empleo de tesauros o la aplicación de filtros metodológicos de búsqueda. 


\section{Revisión crítica de las pruebas}

Una vez recuperadas las mejores pruebas disponibles, es necesario evaluar críticamente esta información. Probablemente, la clave de la metodología de la MBP radica en este punto. La lectura crítica de la información incluye:

1. La valoración de la validez interna, es decir del rigor metodológico.

2. La valoración de la importancia, esto es de la potencial utilidad clínica de los resultados.

3. La valoración de la aplicabilidad.

El EBMWG, publicó a partir de 1993 en la revista JAMA, una serie de trabajos que, bajo el título genérico de: Guías para usuarios de la literatura médica ("Users' guides to the medical literatu$\mathrm{re}^{\prime 27}$ ), enseñan al clínico a valorar críticamente artículos sobre tratamiento o prevención, pruebas diagnósticas, efectos nocivos, pronóstico, revisiones sistemáticas, análisis de decisiones, guías de práctica clínica, etc. Estas guías están disponibles en Internet en la página del "Centre for Health Evidence" 28 .

Aplicación de los resultados en la práctica clínica

La aplicación de los resultados de los estudios investigación clínica al paciente individual no es nunca inmediata. Es en este momento de la toma de decisiones, cuando deben integrarse los tres pilares básicos de la MBP: la experiencia clínica del médico, las mejores pruebas externas disponibles y las preferencias del paciente.

\section{CRÍTICAS A LA MEDICINA BASADA EN PRUEBAS}

El modelo de la MBP ha obtenido una calurosa acogida y ha experimentado, en sus diez años de existencia, una notable difusión, pero también ha recibido fuertes críticas y objeciones que es conveniente reseñar.

En primer lugar, como ya se ha comentado, se ha criticado la pretensión del EBMWG de que su modelo de MBP sea considerado como un cambio de paradigma de la medicina.

Se ha argumentado que este nuevo modelo de medicina, no aporta en realidad novedades y que "es algo que ya se está haciendo"17. Sin embargo, la realidad es que a pesar de los esfuerzos, los clínicos tienen grandes dificultades para mantenerse al día. Los inspiradores de la MBP aportan cinco razones para explicar la necesidad de un nuevo enfoque en la enseñanza y la práctica de la medicina:

1. Permanentemente están surgiendo nuevos tipos de "evidencias" que, cuando las conocemos y las comprendemos, crean cambios importantes y frecuentes en la forma de cuidar a nuestros pacientes.

2. Aunque necesitamos estas nuevas evidencias a diario, no solemos ser capaces de conseguirlas.

3. Como consecuencia de lo anterior, tanto nuestra actualización de conocimientos como nuestro rendimiento clínico se deterioran con el tiempo.

4. El intento de vencer la entropía clínica mediante los programas tradicionales de educación médica continua no mejora nuestro rendimiento clínico.

5. Se ha demostrado que un enfoque distinto del aprendizaje clínico mantiene al día a quienes lo practican ${ }^{10}$.

Otra de las principales críticas se refiere al concepto de prueba o "evidence", que se maneja. El problema podría resumirse así: Medicina Basada en Pruebas sí; pero ¿en qué pruebas? ${ }^{29}$. Se ha atribuido a la MBP un interés exclusivo por los ensayos clínicos controlados aleatorios y por los meta-análisis de ensayos controlados. Los propios promotores de la MBP han salido al paso de estas acusaciones, afirmando que lo que se pretende en realidad es encontrar, en cada caso, el tipo de estudio más adecuado, dependiendo de la pregunta clínica que se quiera contestar. Para evaluar la validez de una prueba diagnóstica se deben emplear ensayos transversales, en los que se someta a los pacientes tanto a la prueba que se quiere analizar como a un estándar, patrón de referencia o prueba confirmatoria. Para evaluar el pronóstico se deben buscar estudios longitudinales de seguimiento de cohortes. Para determinar si un determinado agente es el responsable del desarrollo de una enfermedad se emplearán estudios de cohortes o de casos y controles, según la prevalencia de la enfermedad. Es a la hora de evaluar tratamientos, cuando los ensayos clínicos controlados y aleatorios son los estudios que aportan una información más fiable. Si bien en algunas ocasiones no es posible realizar 
este tipo de ensayos por razones técnicas o éticas y es necesario recurrir a estudios observacionales o cuasiexperimentales para obtener las mejores pruebas ${ }^{17}$. Sin embargo la idea de establecer una clasificación jerárquica de la "evidencia" científica en función del diseño de estudio empleado para obtenerla, no es compartida por todos. Los ensayos clínicos controlados y aleatorios, tienen una gran validez interna y miden adecuadamente la eficacia de los tratamientos, pero no miden la efectividad ni la eficiencia de los mismos y presentan por ello, limitaciones en su aplicación a la práctica clínica, validez externa. Por ello se ha propuesto que la MBP debería de incorporar otro tipo de pruebas, como las que suministran los estudios naturalísticos (efectividad), los estudios de evaluación económica (eficiencia), los estudios cuantitativos (calidad de vida), etc. ${ }^{29}$.

Otro tipo de críticas realizadas a la MBP hace referencia a los aspectos éticos de la relación médico-paciente. Se ha afirmado que la implantación de una MBP podría conducir a la sustitución del juicio clínico por una alianza de administradores y tecnócratas que influyese sobre millones de decisiones clínicas sin tener que dar cuenta de ellas ${ }^{30}$. En realidad, la metodología de la MBP eleva la autonomía individual del médico ante el juicio de las fuentes, lo que conlleva a resaltar el papel de la libertad clínica ${ }^{9}$. Se ha acusado a la MBP de estar al servicio de una política de recorte de gastos, que limita la libertad del clínico. La reducción del gasto sanitario no forma parte de los objetivos de esta corriente de pensamiento. Aunque indirectamente, es probable que una medicina basada en hechos científicamente probados suponga un ahorro en el empleo de técnicas diagnósticas inapropiadas y reduzca el uso de tratamientos de dudosa o escasa eficacia terapéutica, pero sin duda, conducirá a una mejor utilización de los recursos económicos ${ }^{17}$.

Por último, se ha argumentado también la dificultad o imposibilidad de los clínicos para seguir este modelo de autoaprendizaje, tanto por la falta de tiempo generada por la presión asistencial, como por la falta de conocimientos sobre bibliografía, estadística y epidemiología clínica. Se ha apuntado con ironía, que la aplicación sis- temática de la MBP podría devenir en un movimiento migratorio masivo de los médicos desde la cabecera del enfermo al teclado del ordenador y a la biblioteca ${ }^{31}$. Sin embargo, este nuevo esquema de aprendizaje y actualización permite un aprovechamiento más eficaz del tiempo dedicado al estudio, concretando el objetivo de las búsquedas y obteniendo los estudios de mayor calidad. Es cierto que la práctica de esta metodología requiere la adquisición de una serie de habilidades, tanto en la búsqueda de información como en la valoración crítica de los trabajos recuperados, pero es cierto también que cada vez están apareciendo más herramientas que facilitan la búsqueda y ofrecen resúmenes o revisiones sistemáticas, como la Colaboración Cochrane o las revistas secundarias basadas en la "evidencia".

\section{GRUPO DE TRABAJ O EN UROLOGÍA BASADA EN PRUEBAS DE LA ASOCIA- CIÓN MURCIANA DE UROLOGÍA}

La Asociación Murciana de Urología ha promovido, a través de su vocalía científica, la creación del "Grupo de trabajo en Urología Basada en Pruebas de la Asociación Murciana de Urología", que se constituyó en marzo de 2002, como un grupo estable de urólogos, miembros de la Asociación, interesados en el estudio y la práctica de la nueva metodología de la MBP.

Los objetivos de este Grupo de Trabajo son:

1. La autoformación en la metodología y herramientas de la MBP y su aplicación a la Urología.

2. La práctica de la Urología Basada en Pruebas mediante el desarrollo de: un archivo de preguntas clínicas, un archivo de Temas Valorados Críticamente, revisiones sistemáticas, meta-análisis...

3. Difusión de la metodología de la MBP y de los documentos elaborados por el Grupo de Trabajo.

4. Colaboración con otras entidades y grupos nacionales e internacionales, que trabajen en el ámbito de la MBP.

El presente artículo se enmarca en el deseo de este Grupo de Trabajo de difundir el concepto y la metodología de la Medicina Basada en Pruebas en la especialidad de Urología. 


\section{REFERENCIAS}

1. EVIDENCE-BASED MEDICINE WORKING GROUP.: Evidence-based medicine. A new approach to teaching the practice of medicine. JAMA 1992; 268 (17): 2420-2425.

2. EVIDENCE-BASED MEDICINE WORKING GROUP.: La medicina basada en la evidencia. Un nuevo enfoque para la docencia de la práctica de la medicina. JAMA (ed. esp.) 1997: 15-21

3. GUYATT GH.: Evidence-based medicine. ACP Journal Club 1991; 114: A16.

4. REAL ACADEMIA ESPAÑOLA.: Diccionario de la Lengua Española. Vigésimo segunda edición. 2001. http://rea.es.

5. PUIGDOLLERS COLÓS JM.: Precisiones terminológicas a la medicina basada en la evidencia. Med Clin (Barc) 1999; 113: 79.

6. SANZ RUBIALES A, DEL VALLE RIVERO ML, GARAVÍS VICENTE M.: Medicina basada en la evidencia: ¿no hay una traducción mejor?. Med Clin (Barc) 1999; 112: 119.

7. CUENA BOY R, CUENA BOY MI.: Medicina basada en la evidencia: un caso de pereza lingüística. Med Clin (Barc).

8. GOL FREIXA JM.: Bienvenidos a la medicina basada en la evidencia. En: la medicina basada en la evidencia. Guías del usuario de la literatura médica. JAMA (ed. esp.) 1997: 5-14.

9. GOL-FREIXA JM, DEL LLANO SENAARIS JE.: El fenómeno de la "medicina basada en la evidencia". Med Clin 1999; 112 (Supl 1): 3-8.

10. SACKETT DL, RICHARDSON WS, ROSEMBERG W, HAYNES RB.: Medicina Basada en la Evidencia. Cómo ejercer y enseñar la MBE. Churchill Livingstone, Madrid 1997.

11. LAVERA CRAWLEY.: Medicina basada en la evidencia: un nuevo paradigma para el paciente. En: Evidence-Based Medicine Working Group. La medicina basada en la evidencia. Guías del usuario de la literatura médica. Barcelona: JAMA (ed. esp.), Doyma 1997: 22-23.

12. CHAMORRO A, ALONSO P, ARRIZABALAGA J, CARNÉ X, CAMPS V.: Luces y sombras de la medicina basada en la evidencia: el ejemplo del accidente vascular cerebral. Med Clin (Barc) 2001: 116: 343349.

13. MARIÓN BUEN J, PEIRÓ S, MÁRQUEZ CALDERÓN S, MENEU DE GUILLERNA R.: Variaciones en la práctica médica: importancia, causas e implicaciones. Med Clin (Bar) 1998; 110: 382-390.

14. ELLIS J, MULLIGAN I, ROWE J, SACKETT DL.: Impatient general medicine is evidence based. Lancet 1995; 346: 407-410.
15. What proportion of healthcare is evidence based? Resource Guide. http://www.shef.ac.uk/ scharr/ ir/percent.html

16. COCHRANE AL.: 1931-1971: a critical review, with particular reference to the medical profession. In: Medicines for the year 2000. London: Office of Health Economics 1979: 1-11.

17. SACKETT DL, ROSENBERG WM, MUIR GRAY JA, HAYNES RB, RICHARDSON WS.: Evidence based medicine: what it is and isn't. Br Med J 1996; 312: 71-72.

18. EMANUEL EJ, EMANUEL LL.: Four models of the physician-patient relationship. JAMA 1992; 267: 2221-2226.

19. BROWMAN G, GÓMEZ DE LA CÁMARA A, HAYNES B, JADAD A, GABRIEL R.: Herramientas para la práctica de la medicina basada en la evidencia (y II). Desarrollo de guías de práctica clínica basadas en la evidencia de abajo-arriba. Med Clin (Barc) 2001; 116: 267-270.

20. PUBLIC LIBRARY OF SCIECE.: http://www.publiclibraryofscience.org/

21. PUBMED CENTRAL.: An archive of life science journals. http://www.pubmedcentral.nih.gov/

22. COSTE OLASAGASTI JL.: Evaluación de tecnologías médicas basada en la evidencia. http://www. isciii.es /aets / drvisapi.dll? MIval=cw-usr-viewSHTML\&ID $=1005$

23. COLABORACIÓN COCHRANE: Tríptico de difusión de la Colaboración Cochrane. http://www.cochrane.es/Download/Files/triptic.doc

24. Welcome to CASP. http://www.phru.org.uk/ casp/

25. Programa de habilidades en lectura crítica. España. http://www.redcaspe.org/

26. The CASP international Network. http://www.caspinternational.org.uk/spanish.htm

27. GUYATT GH.: Users' guides to the medical literature. JAMA 1993; 270: 2096-2097.

28. Users' guides to Evidence-Based Practice. Centre for Health Evidence. http://www.cche.net/usersguides/main.asp

29. SOTO J.: Medicina basada en la evidencia: pero ¿en qué evidencia? Med Clin (Barc) 1998; 111: 539-541.

30. CARLTON BG, MILES A.: The rise an fall of EBM. QJM 1988; 5: 371-374.

31. POZO-RODRÍGUEZ F.: La medicina basada en la evidencia. Una perspectiva desde la clínica. Med Clin (Barc) 1999; 112: 12-16.

Dr. C. Tello Royloa

Avda. de Europa, 9 - 2을

30007 Murcia

(Trabajo recibido el 7 noviembre de 2002) 